Claremont Colleges

Scholarship@Claremont

6-15-1978

\title{
The Kinetics of Solvent Reorientation in Hydroxylated Solvents From the Exciting- wavelength Dependence of Chromophore Emission Spectra
}

John Milton

Claremont McKenna College; Pitzer College; Scripps College

Robert M. Purkey

William C. Galley

McGill University

\section{Recommended Citation}

Milton, John G., Robert M. Purkey, William C. Galley. "The Kinetics of Solvent Reorientation in Hydroxylated Solvents From the Exciting-Wavelength Dependence of Chromophore Emission Spectra." Journal of Chemical Physics 68.12 (1978): 5396-5404. DOI: $10.1063 / 1.435704$ 


\title{
The kinetics of solvent reorientation in hydroxylated solvents from the exciting-wavelength dependence of chromophore emission spectra
}

\author{
John G. Milton, Robert M. Purkey, and William C. Galley \\ Department of Chemistry, McGill University, Montreal, Quebec, Canada H3C 2 K6 \\ (Received 21 October 1976) \\ The disappearance of the exciting-wavelength dependence of the phosphorescence spectra of polar, \\ aromatic chromophores in supercooled glycol-water mixtures is utilized to monitor the kinetics of solvent \\ reorientation. Reorientation times in the nanosecond to second range are obtained for $(3: 2 v / v)$ \\ glycerol-water and $(1: 1 v / v)$ ethylene glycol-water at $140-240^{\circ} \mathrm{K}$. The results suggest that the \\ process is one involving a cluster of solvent molecules and in which the chromophore plays a relatively \\ passive role. Steady-state data and direct measurements of phosphorescence shifts as a function of time \\ indicate that the solvent reorientation process is nonexponential in nature. The decay function derived \\ from the temperature dependence of the steady state data is consistent with the decays observed directly \\ as a function of time. Interpretation of this nonexponential decay in terms of a summation of rate \\ processes leads to a distribution dominated by two reorientation rate constants. The relative contributions \\ of the slow and faster reorientation rate constants in addition to their activation parameters differ for the \\ glycerol-water and ethylene glycol-water mixtures.
}

\section{INTRODUCTION}

While the emission spectra of aromatic molecules in fluid solution is normally independent of the wavelength of excitation, a shift in the spectra with exciting wavelength has been reported for polar aromatic molecules in solvents which are polar and rigid. ${ }^{1-11}$ In an earlier publication $^{1}$ the role of solvation-site heterogeneity at the basis of this effect was emphasized. The excitingwavelength dependence was envisaged as arising as a consequence of the photoselection of molecules occupying differing solvation geometries and hence possessing differing transition energies. Similar mechanisms have been discussed by Rubinov and Tomin ${ }^{2}$ and Itoh and Azumi. ${ }^{3}$

The dynamic nature of the exciting-wavelength dependence which follows quite naturally as a consequence of a solvation-site heterogeneity was evidenced by the observation ${ }^{1}$ that (1) the exciting-wavelength dependence of emission spectra was observed to be temperature dependent and vanished as the solvent became sufficiently mobile for randomization of the solvation sites to occur, and (2) the temperature at which emission spectra became independent of exciting wavelength was found to depend on the excited-state lifetime of the chromophore; for a given molecule occurring at a much lower temperature for phosphorescence than for fluorescence. Thus, the exciting-wavelength dependence of emission spectra depends on the interplay between the rate at which an excited state population decays and the rate at which solvent molecules reorganize. The present communication describes the quantification of the above observations to provide information on the kinetics of solvent reorientation processes in glycol-water mixtures.

\section{EXPERIMENTAL}

2-Aminobenzoic acid (Eastman Organic Chem.), 2amino-5-bromobenzoic acid (K \& K Laboratories), 2 amino-5-chloro-benzoic acid (Matheson, Coleman \& Bell), 2-amino-5-iodobenzoic acid (Aldrich Chem.), 5-bromoindole ( $\mathrm{K} \& \mathrm{~K}$ Laboratories), 5-chloroindole (Aldrich Chem.), and indole ( $\mathrm{K} \& \mathrm{~K}$ Laboratories) were recrystallized in the dark from alcohol-water before use. Proflavin (K \& K Laboratories) and 8-anilino-1naphthalene-sulfonic acid (Fisher) were of the highest purity available from the manufacturer and were used without further purification. Ethylene glycol, "Chromatoquality," and glycerol, "Spectrograde," obtained from Matheson, Coleman, and Bell were checked for emission impurities prior to use. Only solvents for which the ratio of the signal arising from chromophore emission to solvent emission was better than 100 were used. Glasses were prepared from $3: 2 v / v$ glycerolwater (GW) or $1: 1 v / v$ ethylene glycol-water (EGW) solutions. The densities of the pure solvents were used to calculate the appropriate weight of each solvent to be added to give the equivalent volume to volume solution. The final concentration of the chromophore was $3 \times 10^{-6} \mathrm{M}$ for proflavin and $5 \times 10^{-4} M$ for all others. Preliminary experiments had shown that at these concentrations the solutions were free from solute aggregation. Solutions were placed in $4 \mathrm{~mm}$ i. d. suprasil quartz tubes for observation of the emission.

Spectra were recorded on an instrument which has been described previously ${ }^{12}$ and were uncorrected for instrument sensitivity. With the exception of 2-amino5-bromobenzoic acid and 2-amino-5-iodobenzoic acid, phosphorescence lifetimes were obtained either by interrupting the exciting beam and following the emission decay on a Moseley $X-Y$ recorder, or by recording the phosphorescence decay on an oscilloscope following a single exciting pulse of about $100 \mathrm{msec}$ achieved by rotating the phosphorescence choppers through one complete dark-light-dark cycle. The lifetimes of the phosphorescence of 2-amino-5-bromobenzoic acid and 2amino-5-iodobenzoic acid which were too short to be monitored in this manner, were measured from the decay of the emission excited with a pulsed nitrogen laser $^{13}$ at $337 \mathrm{~nm}$.

Phosphorescence emission shifts were measured as 
TABLE I. Transition temperature $T_{1 / 2}$ observed for chromophores with differing lifetimes.

\begin{tabular}{|c|c|c|c|c|c|c|}
\hline & Code & Emission & $\begin{array}{l}\lambda_{\text {oxcitat ion }} \\
\text { (nm) }\end{array}$ & $\begin{array}{l}\sigma_{\max } \\
(\mathrm{nm})\end{array}$ & $\begin{array}{l}T_{1 / 2} \\
(\mathrm{~K})\end{array}$ & $\tau(\mathrm{sec})^{2}$ \\
\hline Indole & 1 & $\mathrm{P}$ & 280,295 & 1.2 & 158.0 & 5.0 \\
\hline Proflavin & 2 & $\mathbf{P}$ & 436,460 & 11.0 & 159.0 & 1.4 \\
\hline Anthranilate $^{b}$ & 3 & $\mathrm{P}$ & 358,368 & 3.9 & 160.0 & 1.1 \\
\hline $\mathrm{Cl}$-indole & 4 & $\mathrm{P}$ & 283,308 & 1.2 & 160.5 & 0.8 \\
\hline $\mathrm{Cl}$-anthranilate ${ }^{\mathrm{b}}$ & 5 & $P$ & 358,368 & 3.5 & 161.0 & 0.35 \\
\hline $\mathrm{Br}$-indole & 6 & $\mathrm{P}$ & 290,302 & 0.9 & 164.7 & 0.025 \\
\hline $\mathrm{Br}$-anthranilate ${ }^{\mathrm{b}}$ & 7 & $\mathrm{P}$ & 360,380 & 4.1 & 165.3 & 0.007 \\
\hline I-anthranilate ${ }^{b}$ & 8 & $\mathrm{P}$ & 360,370 & 1.8 & 169.5 & 0.0004 \\
\hline 8-ANS & 9 & $F$ & 380,415 & 12.8 & 199.5 & $15 \times 10^{-9 c}$ \\
\hline Indole & 10 & $\mathrm{~F}$ & 280,295 & 10.3 & 206.5 & $5 \times 10^{-9 d}$ \\
\hline Proflavin & 11 & $\mathrm{~F}$ & 436,460 & 3.8 & 203.0 & $4.5 \times 10^{-90}$ \\
\hline
\end{tabular}

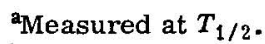

Samples contained $0.02 M$ acetate, $p H 4.0$.

${ }^{2}$ Lifetime reported by Anderson and Weber ${ }^{14}$ in aqueous bovine serum albumin solution.

${ }^{\mathrm{d}}$ Lifetime reported by Walker et al. ${ }^{15}$ in water at $283 \mathrm{~K}$.

${ }^{e}$ Lifetime reported by Chen et al. ${ }^{16}$ in buffer at room temperature.

the difference in either the position of the emission maximum or the position of one-half intensity measured on the blue edge of the emission spectrum. Fluorescence emission shifts were measured on the red edge of the emission spectrum in order to avoid the exciting beam. Phosphorescence spectra of 5-bromoindole, 2amino-5-bromobenzoic acid, and 2-amino-5-iodo benzoic acid were measured without phosphorescence choppers and the emission shifts measured as for fluorescence spectra. The parameters calculated in the following sections were independent of the measurement used. Table I gives the exciting wavelengths used and the shift observed at $77 \mathrm{~K}$ for the chromophores used. These shifts were reproducible to within $0.1 \mathrm{~nm}$. In measuring chromophore emission shifts, the two exciting wavelengths were chosen solely on the basis of obtaining a measurable emission shift.

Sample temperatures were varied by adjusting the flow of cold nitrogen gas through a quartz Dewar housing the sample tube. The usual rate of temperature change was $0.75 \pm 0.25 \mathrm{deg} / \mathrm{min}$. Sample temperatures were monitored with a copper-constantan thermocouple placed in a second solvent-containing tube next to the sample tube. Hysteresis effects were not observed; however, in general experiments were carried out as a function of increasing temperature only to avoid crystallization of the solution which tended to occur with slowly decreasing temperatures.

\section{RESULTS AND DISCUSSION}

\section{A. The variation of the exciting-wavelength dependence of emission spectra with temperature and lifetime}

The exciting-wavelength dependence of the emission spectra of a number of chromophores in GW appears as a function of temperature in Fig. 1. For each chromophore the shift in the emission spectra $\sigma$ obtained by exciting at two different wavelengths has been normalized to the value $\sigma_{\max }$ observed at $77 \mathrm{~K}$. The temperature range over which a loss of exciting -wavelength dependence occurs is observed to be a function of the excitedstate lifetime of the chromophore, $\tau$. As $\tau$ becomes shorter, the decrease in $\sigma / \sigma_{\mathrm{max}}$ is observed at warmer temperatures.

The loss in exciting wavelength dependence with increasing temperature in Fig. 1 contains a nonkinetic contribution. This is most apparent on the low temperature side on the transition where, for example, the changes in $\sigma / \sigma_{\max }$ for proflavin phosphorescence and for 8 -ANS fluorescence are the same despite the $10^{8}$-fold

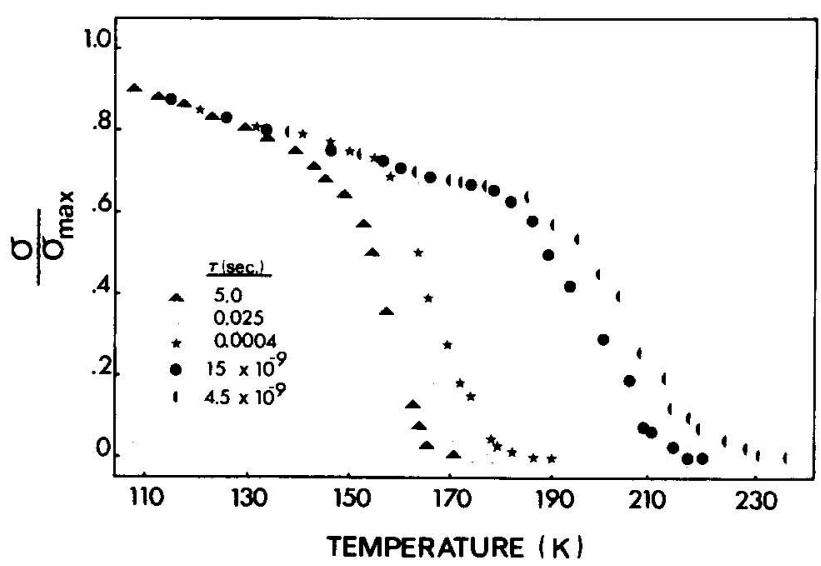

FIG. 1. Loss of exciting-wavelength dependence of emission spectra for indole (4), 2-amino-5-bromo benzoic acid (o), and 2-amino-5-iodo-benzoic acid $(\star)$ phosphorescence and 8ANS $(\bullet)$ and indole $(1)$ fluorescence as a function of temperature in glycerol $-\mathrm{H}_{2} \mathrm{O}(3: 2 v / v)$. 


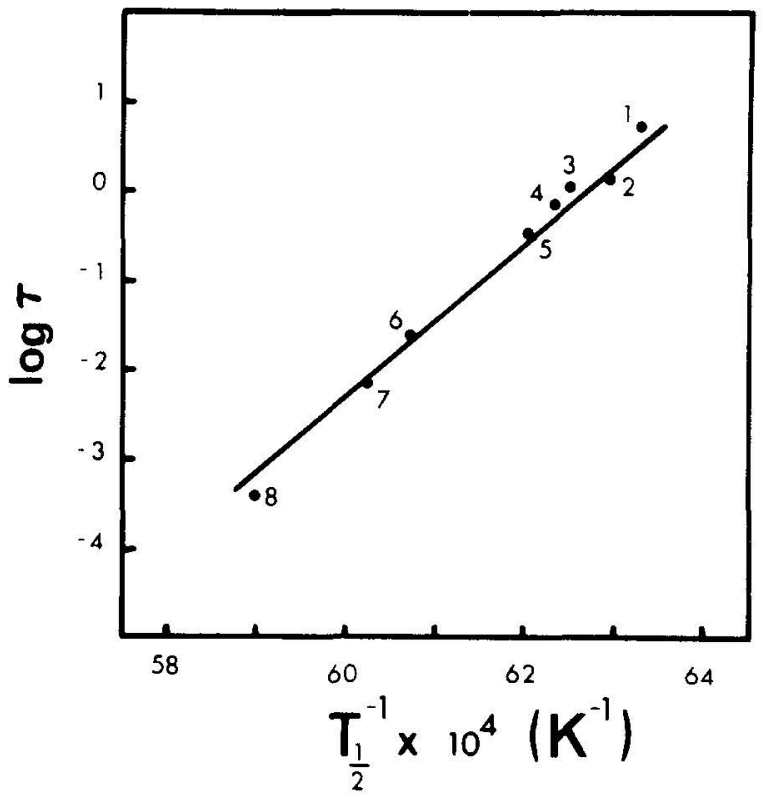

FIG. 2. Plot of the logarithm of the chromophore phosphorescence excited-state lifetime, $\tau$, as a function of $T_{1 / 2}^{-1}$. Numbers refer to the chromophores listed in Table I.

difference in their excited state lifetimes. In that our present concern is with the contribution arising from dynamic processes an empirical correction was made for this lifetime independent contribution. This correction as indicated in the Appendix had little influence on the results. The lifetime-dependent portion was interpreted in terms of the dynamics of solvent reorientation.

Transitions such as those shown in Fig. 1 can be characterized by the temperature $T_{1 / 2}$ at which $\sigma$ $=\frac{1}{2} \sigma_{\max }$. Table I provides the values of $T_{1 / 2}$ deter mined for a number of chromophores with differing lifetimes $\tau$, in glycerol-water $(3: 2 v / v)$. The linear relationship between $T_{1 / 2}^{-1 / 2}$ and $\tau$ for chromophores of quite different structure and polarity shown in Fig. 2 provides evidence that the chromophore itself cannot be a dominating parameter in the kinetics of reorganization of the solvent molecules about the chromophore. If $T_{1 / 2}$ is taken as the temperature at which the time for solvent reorientation equals $\tau$, then the linear relationship between $T_{1 / 2}^{-1}$ and $\log \tau$ suggests that the reorientation process can be described in terms of a rate constant with an Arrhenius temperature dependence of the form

$$
k=A \exp \left(-E_{a} / R T\right),
$$

where $A$ and $E_{a}$ are the pre-exponential factor and activation energy, respectively. The values of $\log A$ and $E_{a}$ determined from this plot are $51.7 \pm 1.3$ and 38.0 $\pm 0.3 \mathrm{kcal} / \mathrm{mole}$, respectively.

Interpreting the pre-exponential factor in accordance with absolute rate theory in terms of an activation entropy $\Delta S^{\dagger}$ from the relation

$$
A=\frac{e k T}{h} \exp \left(\Delta S^{\dagger} / R\right)
$$

in which $h, e$, and $k$ are Planck's constant, the base of natural logarithms, and the Boltzmann factor, respectively, leads to an activation entropy of $178 \mathrm{cal} \mathrm{mole}^{-1}$ - $\operatorname{deg}^{-1}$. Exceedingly large activation entropies are characteristic of many relaxation processes in hydroxylated solvents and have been interpreted as indicating that a group of molecules rather than a single molecule represents the reorienting unit. ${ }^{17}$ The cooperative nature of the process involving the simultaneous reorgannization of a number of molecules about the chromophore is probably at the basis of the observation that the chromophore appears to be playing a passive role. This does not imply that chromophore-solvent interactions which differ from one chromophore to the next are not present, but only that the dynamics of solvent reorganization involve a sufficiently large cluster of molecules so that these variations do not constitute sizeable perturbations.

\section{B. Comparison of the data with a model involving a single solvent-reorientation rate constant}

The characterization of the data in Fig. 2 in terms of an Arrhenius temperature dependence suggests that the reorganization of solvent geometries can be described in terms of a rate constant with the activation parameters given above. This implies that distinct solvation geometries selected at $t=0$ would undergo randomiza tion in the form

$$
\phi(t, T)=\exp [-k(T) t]
$$

where $\phi(t, T)$ is a function normalized at $t=0$ which is a measure of the difference between solvation geometries and $k(T)$ is the reorientation rate constant. In the steady state experiments described above, the decay of the orientation of the solvation geometries is not measured directly as a function of time. At all instances in time there are populations of chromophores that have been in the excited state for varying periods of time. The relevant function is therefore the time-averaged value of $\phi(t, T)$, which is defined as

$$
\overline{\phi(t, T)}=\int_{0}^{\infty} f(t) \phi(t, T) d T,
$$

where $f(t)=\exp (-t / \tau) / \tau$ represents the fraction of molecules in the steady state population that have existed in the excited state for a time $t$. Setting $\overline{\phi(t, T)}=\sigma / \sigma_{\max }$ and utilizing Eqs. (3) and (4), we find that the loss in exciting wavelength dependence in a steady state experiment is given by

$$
\sigma_{\mathrm{max}} / \sigma=1+k T
$$

and is now a function of $\tau$ rather than time. Taking the logarithm of Eq. (5) and incorporating an Arrhenius temperature dependence for the reorientation rate constant of the form of Eq. (1) yields

$$
\log \left(\frac{\sigma_{\max }}{\sigma}-1\right)=\log A \tau-E_{\mathrm{a}} / 2.3 R T
$$

the temperature of the midpoint of the transition where $\sigma_{\max } / \sigma=2$,

$$
\log \tau=\frac{E_{a}}{2.3 R T_{1 / 2}}-\log A,
$$




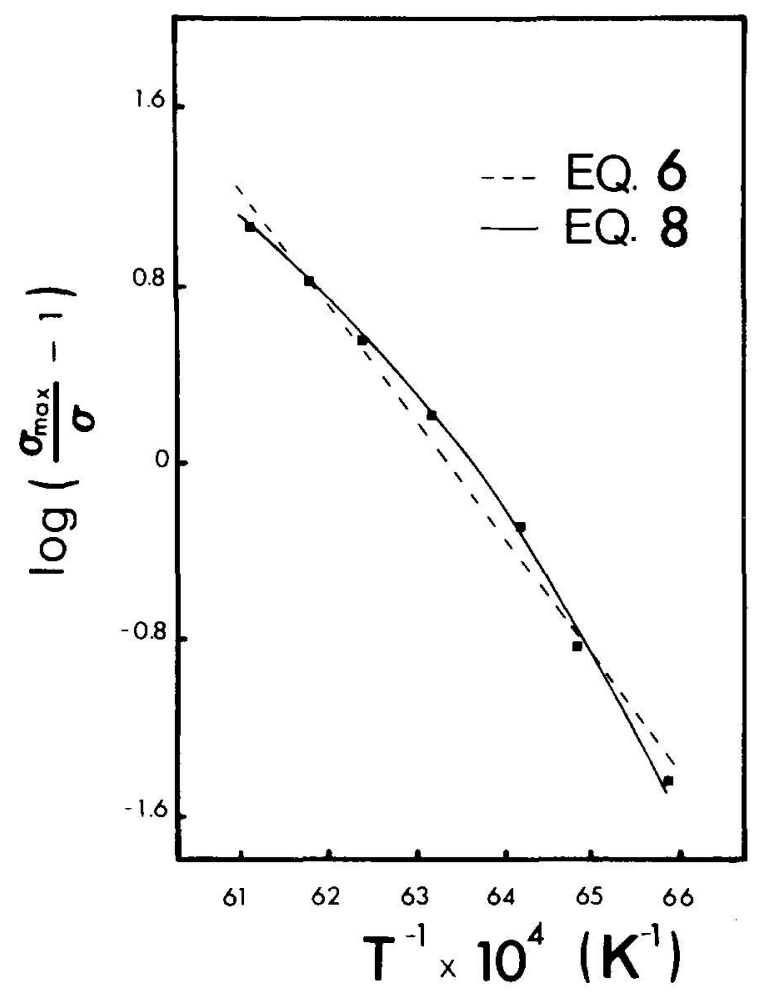

FIG. 3. Plot of $\log \left(\sigma_{\max } / \sigma-1\right)$ vs $T^{-1}$ for proflavin phosphorescence in glvcerol-water $(3: 2 v / v)$. The experimental points are taken from five trials. Dotted line was determined by a linear regression analysis and solid line was calculated from

$$
\log \left(\frac{\sigma_{\max }}{\sigma}-1\right)=\log \left[(1+\tau)^{\alpha}-1\right]
$$

It follows from Eq. (6) that for any chromophore characterized by a lifetime $\tau$, a plot of $\log \left[\left(\sigma_{\max } / \sigma\right)-1\right]$ vs $T^{-1}$ should be linear and in addition yield values of $A$ and $E_{a}$ equal to those obtained by plotting the midpoints of the transitions of a number of chromophores with differing lifetimes as in Fig. 2. This hypothesis is tested in Fig. 3, which shows a plot of $\log \left(\sigma_{\max } / \sigma-1\right)$ vs $T^{-1}$ for proflavin phosphorescence in GW. The values of $\log A$ and $E_{a}$ which would be obtained if a linear relationship is assumed (dotted line) are $33.5 \pm 1.7$ and 24.5 $\pm 1.1 \mathrm{kcal} / \mathrm{mole}$, respectively. In view of the foregoing discussion two inconsistencies can be seen: (1) The relationship is not linear, and (2) The values of $\log A$ and $E_{a}$ are smaller than those obtained from an evaluation of the data given in Fig. $2(51.7$ and $38.0 \mathrm{kcal} / \mathrm{mole}$, respectively). Thus, Eqs. (6) and (7) do not provide a self-consistent description of the observations. Similar observations were obtained with all chromophores examined and with pure glycerol, ethylene glycol $-\mathrm{H}_{2} \mathrm{O}$, and propylene glycol $-\mathrm{H}_{2} \mathrm{O}$ solvents. It is clear that the solvent reorientation in these liquids monitored with the present approach cannot be described in terms of a single reorientation time over the temperature range investigated in these experiments. Dielectric relaxation studies on these liquids have also yielded this conclusion. ${ }^{22,23}$

\section{An empirical description of solvent reorientation in glycol-water}

While the data in Fig. 3 cannot be accounted for in terms of $\mathrm{Eq}$. (5), an empirical equation can be found which will fit the data. This equation has the form

$$
\sigma_{\mathrm{max}} / \sigma=\left(1+k_{0} \tau\right)^{\alpha},
$$

where $\alpha$ is an adjustable parameter, $0<\alpha<1$, and $k_{0}$ is a reorientation rate constant. By utilizing the graphical analysis described in the Appendix, the data in Fig. 3 yield values of $\alpha, \log A$, and $E_{a}$ of $0.45,49.2$, and 35.3 $\mathrm{kcal} / \mathrm{mole}$, respectively. The solid line shown in Fig. 3 has been calculated from Eq. (8) with these parameters.

It follows from Eq. (8) that the midpoint of the temperature profile where $\sigma=\frac{1}{2} \sigma_{\max }$ does not quantitatively represent the temperature at which $k_{0}=1 / \tau$. This temperature now depends on $\alpha$ and occurs at the point where $\sigma=\mathbf{2}^{-\alpha} \sigma_{\max }$. In addition we see that a plot of $\log \tau$ vs the reciprocal of the temperature $\left(T_{k_{0}=1 / \tau}\right)$ at which $k_{0}=1 / \tau$ will be linear with slope $E_{a} / 2.303 R$ and inter cept equal to $\log \left(\psi^{\alpha-1}-1\right)-\log A$, where $\psi$ is the value of $\sigma_{\max } / \sigma$ at $T_{k_{0}=1 / \tau}$. Thus, Eq. (8) also predicts linearity of the type observed in Fig. 2.

Figure 4 shows a plot of $\log \tau$ as a function of the reciprocal of $T_{k_{0}=1 / \tau^{*}}$. From this plot (solid line) we obtain values for $\log A$ of $46.7 \pm 3.1$ and $E_{a}$ of $34.2 \pm 2.3$ $\mathrm{kcal} / \mathrm{mole}$. Table II gives the values of $\alpha, \log A$, and $E_{a}$ obtained from the evaluation of data of the type shown in Fig. 3 for a variety of chromophores. As can be seen, over a comparable temperature range there is very good agreement between these values of $E_{a}$ and $\log A$ and those obtained from Fig. 4. This observation serves to emphasize the validity of the method over small temperature ranges. Inspection of Fig. 4 indicates that over a much larger temperature interval encompassing temperatures at which reorientation occurs on a nanosecond scale (fluorescence data) this plot is nonlinear. The significance of the temperature dependencies in $\alpha, E_{a}$, and $\log A$ will be examined in a separate communication. The more satisfactory fit of the data afforded by use of Eq. (8) indicates that two parameters, $\alpha$ and $k_{0}$, are required to characterize the sol vent reorientation processes in glycol-water mixtures.

\section{Solvent reorientation decay function}

The observation that the steady state solvent reorientation data was consistent with a relationship of the form of $\mathrm{Eq}$. (8) rather than the simpler form of Eq. (5) implies that the decay function for solvent reorientation is not a simple exponential. The appropriate decay $F(t, T)$, can be determined as the solution of the following integral equation:

$$
\int_{0}^{\infty} f(t) F(t, T) d t=\frac{1}{\left(1+k_{0} \tau\right)^{\alpha}} .
$$

The solution is obtained as the inverse Laplace transform of the right hand side and is found to be $\mathrm{b}^{18}$

$$
F(t, T)=\exp \left(-k_{0} t\right) M\left(1-\alpha, 1, k_{0} t\right)
$$




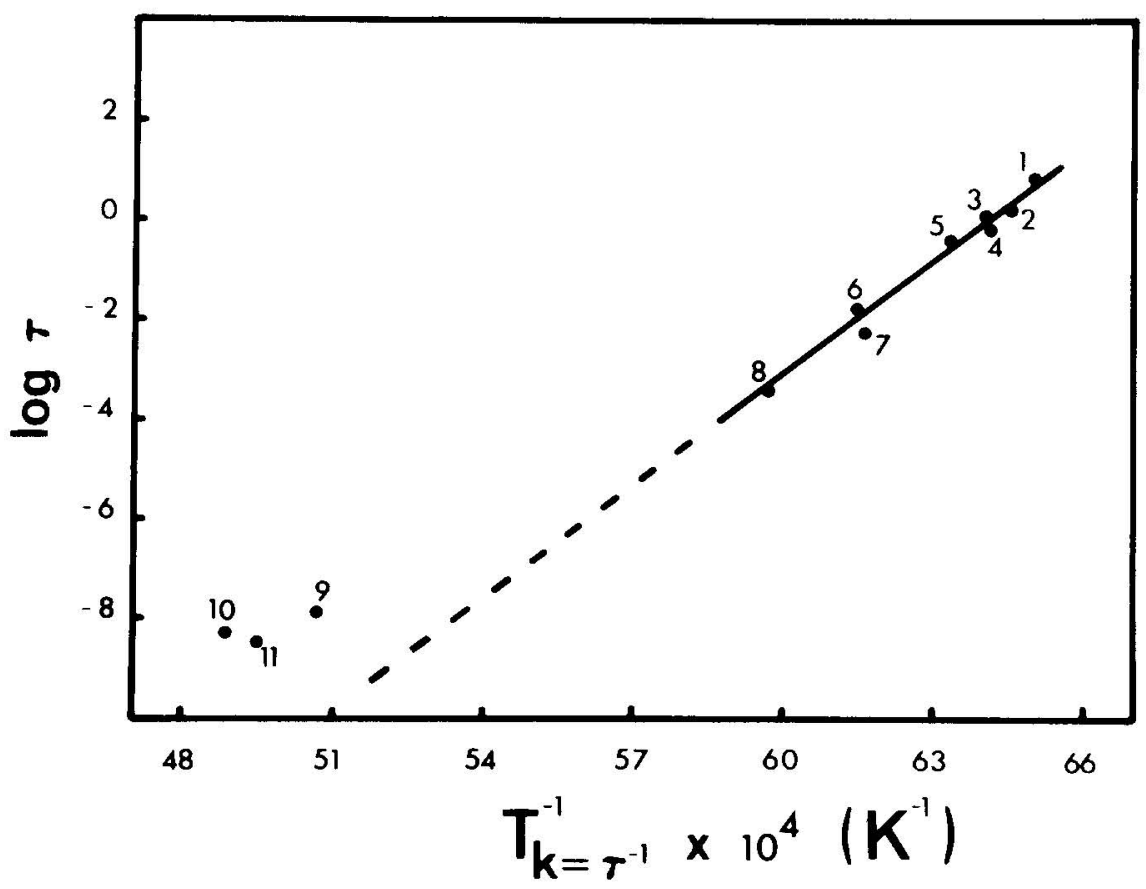

FIG. 4. Plot of the logarithm of the chromophore excitedstate lifetime, $\tau$, as a function of $T_{k_{0}=1 / \tau}^{-1}$. Numbers refer to the chromophores listed in Table I.

where $M\left(1-\alpha, 1, k_{0} t\right)$ is a confluent hypergeometric function. Figure 5 illustrates the shape of $F(t, T)$ as a function of the parameter $\alpha$. It can be seen that Eq. (10) predicts a nonexponential decay and that the degree of nonexponentiality is related to $\alpha$. For all values of $\alpha$, the decay is slower than that of a simple exponential with the same rate constant $k_{0}$, and the more slowly decaying components become increasingly significant as $\alpha$ becomes smaller.

\section{E. The measurement of the time dependence of solvent reorientation}

The solvent reorientation decay function $F(t, T)$ was measured directly from the decay of the emission intensity following an exciting pulse which populated the triplet state of proflavin in a time which was short compared to both the lifetime and the solvent reorientation time $1 / k_{0}$. The experiment was performed at a temper-

TABLE II. Activation parameters associated with solvent reorganization in glycol-water solvents obtained with variety of chromophores.

\begin{tabular}{|c|c|c|c|c|c|c|}
\hline Chromophore & Emission ${ }^{a}$ & $\begin{array}{l}T_{k=1 / \tau} \\
(\mathrm{K})\end{array}$ & $\alpha$ & $\begin{array}{l}E_{a} \\
(\mathrm{kcal} / \mathrm{mole})\end{array}$ & $\log A$ & $\tau(\mathrm{sec})^{\mathbf{b}}$ \\
\hline Indole & $\mathrm{P}$ & 153.9 & 0.45 & $34.8 \pm 5.0$ & $48.3 \pm 5.7$ & 5.4 \\
\hline Proflavin & $\mathrm{P}$ & 155.0 & 0.45 & $35.3 \pm 2.9$ & $49.2 \pm 3.0$ & 1.4 \\
\hline Proflavin & $\mathrm{P}$ & 144.2 & $0.70^{c}$ & $21.4 \pm 1.5$ & $32.7 \pm 1.6$ & 1.4 \\
\hline 2-amino-benzoic acid & $\mathrm{P}$ & 156.3 & 0.45 & $35.1 \pm 2.8$ & $48.7 \pm 3.5$ & 1.1 \\
\hline 5-chloro-indole & $\mathrm{P}$ & 155.8 & 0.45 & $33.6 \pm 5.1$ & $46.9 \pm 6.8$ & 0.8 \\
\hline $\begin{array}{l}\text { 2-amino-5-chloro- } \\
\text { benzoic acid }\end{array}$ & $\mathrm{P}$ & 157.7 & 0.45 & $34.3 \pm 3.6$ & $47.7 \pm 4.1$ & 0.35 \\
\hline 5-bromo indole & $\mathrm{P}$ & 162.9 & 0.45 & $34.6 \pm 3.9$ & $47.7 \pm 5.0$ & 0.025 \\
\hline $\begin{array}{l}\text { 2-amino-5-bromo } \\
\text { benzoic acid }\end{array}$ & $\mathrm{P}$ & 162.3 & 0.45 & $35.0 \pm 2.4$ & $48.9 \pm 3.6$ & 0.007 \\
\hline $\begin{array}{c}\text { 2-amino-5-iodo- } \\
\text { benzoic acid }\end{array}$ & $\mathrm{P}$ & 167.5 & 0.45 & $33.3 \pm 4.1$ & $46.5 \pm 5.0$ & 0.0004 \\
\hline 8-ANS & $\mathrm{F}$ & 197.2 & 0.80 & $19.2 \pm 3.0$ & $29.0 \pm 3.2$ & $15 \times 10^{-9}$ \\
\hline Indole & $\mathrm{F}$ & 204.8 & 0.80 & $16.8 \pm 1.2$ & $26.1 \pm 1.1$ & $5 \times 10^{-9}$ \\
\hline Proflavin & $\mathrm{F}$ & 202.0 & 0.80 & $18.2 \pm 1.5$ & $27.9 \pm 2.0$ & $4.5 \times 10^{-9}$ \\
\hline
\end{tabular}

${ }^{2}$ Phosphorescence (P), fluorescence (F).

${ }^{b}$ Chromophore lifetime measured at $T_{k_{0}=1 / \tau}$; see table $I$.

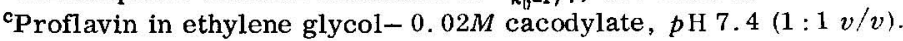




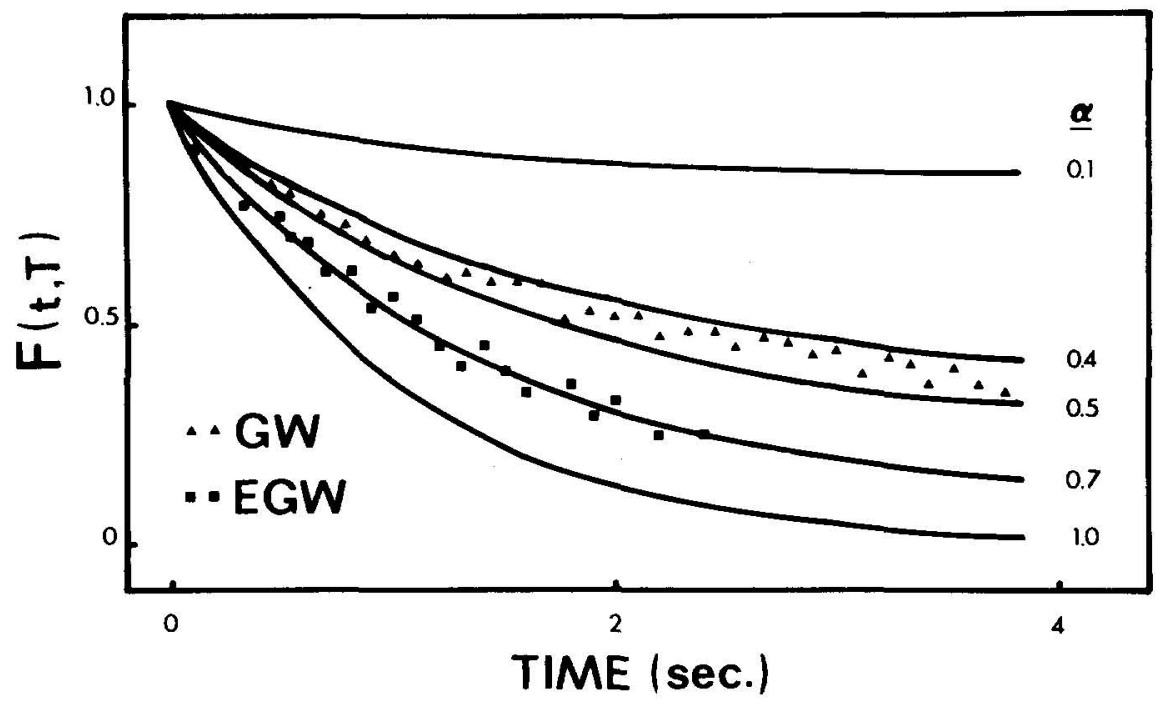

FIG. 5. Decay function for solvent reorientation. Solid lines are calculated from Eq. (10) for $k=1 \mathrm{sec}^{-1}$ and various values of $\alpha$. Experimental points are for proflavin phosphorescence in EGW (ص) at $145 \mathrm{~K}$ and $\mathrm{GW}(\Delta)$ at $156 \mathrm{~K}$. The decay with $\alpha=1$ is an exponential with $k=1 \mathrm{sec}^{-1}$.

ature at which $\tau \approx 1 / k_{0}$. Under these conditions the emission intensity at a given wavelength decays not only as a consequence of the decline in the excited state population but in addition due to the shift in the position of the emission spectrum as a result of the solvent reorientation processes. ${ }^{19-21}$ The phosphorescence decay of proflavin in GW at $580 \mathrm{~nm}$ on the red side of the spectrum and at $535 \mathrm{~nm}$ on the blue side following a 100 msec pulse is shown in Fig. 6. The emission wavelengths were chosen on the basis of the spectra observed as a function of temperature, and the excitation wavelength was one which was observed to produce a "blue spectrum" at low temperatures. The blue edge of the proflavin phosphorescence spectrum observed when solvent randomization has occurred at warmer temperatures is at $535 \mathrm{~nm}$. With the assumption that the spectral shape does not change substantially as a function of time the decline in emission intensity at $535 \mathrm{~nm}$ becomes a measure of the decrease in the shift of the spectrum $\sigma$, once the decay in the emission intensity due to the lifetime of the triplet state has been taken into ac- count. The triplet state lifetime $\tau$ was estimated by interpolation from measurements in the steady state at temperatures just above and below the transition where spectral shifting does not contribute to the decay.

In Fig. 5 the decay function $F(t, T)=e^{t / \tau} \cdot P_{535 \mathrm{~mm}}(t, T)$, where $P_{535 \mathrm{~nm}}(t, T)$ represents the measured phosphorescence intensity at $535 \mathrm{~nm}$, is shown for proflavin in GW and EGW at a temperature which was chosen so that $k_{0}$ $\approx 1 \mathrm{sec}^{-1}$. The decay is nonexponential in both cases and contains a larger contribution of more slowly decaying components for GW than EGW. These features are predicted by the steady state analysis. The values of the parameter $\alpha=0.45,0.6$ (cf. Table II) for GW and EGW, respectively, obtained in the steady state analysis predict that for the same value of $k_{0}, F(t, T)$ should decay more rapidly for EGW than for GW. The decays predicted on the basis of Eq. (10) with $k_{0}=1 \mathrm{sec}^{-1}$, and $\alpha$ $=0.4-0.5$ and 0.6 are seen in Fig. 5 to account for the type of decays observed in the time-dependent experiments.

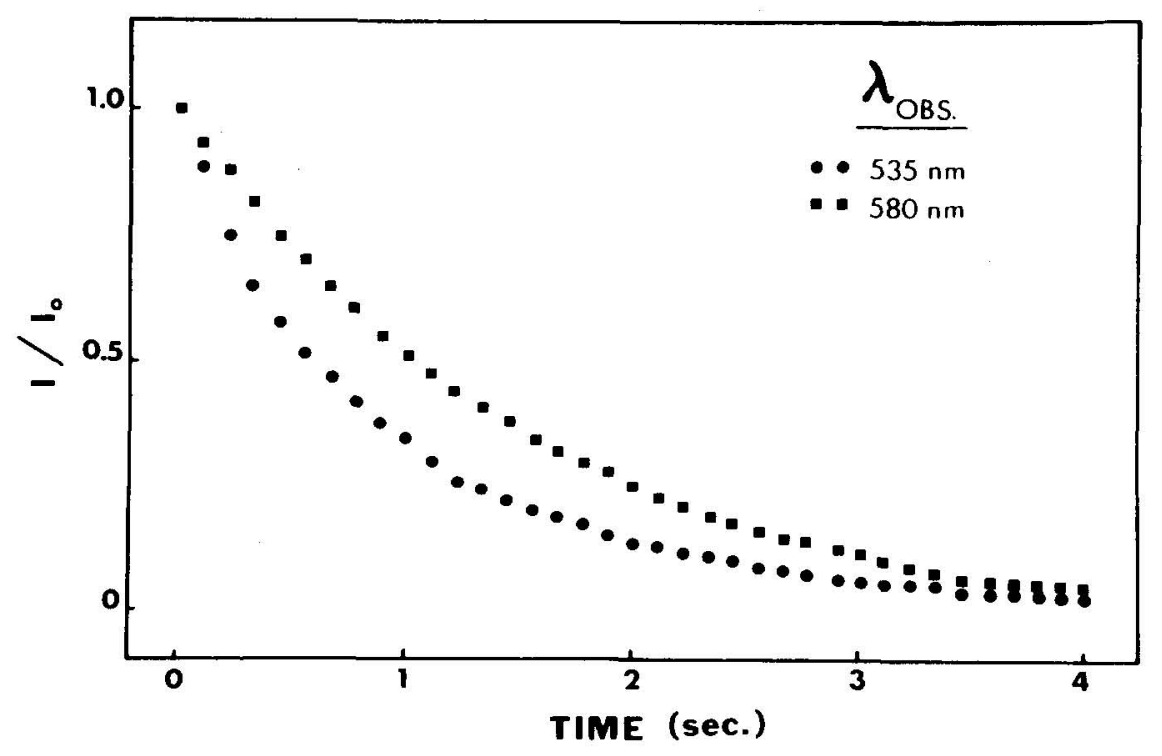

FIG. 6. Decay of the proflavin emission intensity, $I$, in $\mathrm{GW}$ at $156 \mathrm{~K}$ monitored at $535 \mathrm{~nm}(\bullet)$ and $580 \mathrm{~nm}(\bullet)$. Emission intensities are normalized to the intensity recorded at $t=0, I_{0}$. 


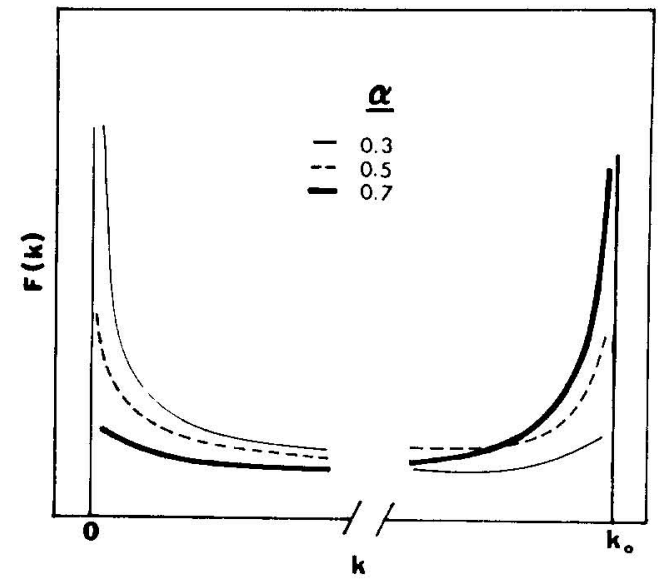

FIG. 7. Distribution function for solvent reorientation given by Eq. (12) as a function of $\alpha$. For the sake of comparison, $F(k)$ has been "normalized" to the sum of the calculated $F(k)$ for each using values of $k$ between 0.01 and 0.99. The light line is for $\alpha=0.3$, the dashed line for $\alpha=0.5$, and the heavy line for $\alpha=0.7$.

The observation of a nonexponential decay function for solvent reorganization about chromophores in these glycol-water mixtures at low temperature is consistent with the earlier findings of nonexponential decay functions for solvent measured from dielectric relaxation methods. ${ }^{22,23}$

\section{F. Distribution of reorientation rate constants}

In the previous sections it was established that solvent reorientation could not be described in terms of an exponentially decaying process. Both the steady state temperature loss in exciting-wavelength dependence and direct measurements of phosphorescence decays as a function of time were consistent with the more complex decay function given by Eq. (10). Since Eq. (8) is empirical in nature the molecular basis of a decay function of the form of Eq. (10) and the physical meaning of the parameters $\alpha, k_{0}$ remains obscure.

If it is assumed that $F(t, T)$ arises as a summation of exponentially decaying processes, it follows that

$$
\int_{0}^{\infty} F(k) e^{-k t} d k=e^{-k_{0} t} M\left(1-\alpha, 1, k_{0} t\right)
$$

where $F(k)$ represents a distribution of exponentially decaying processes or of reorientation rate constants. By again applying the method of inverse Laplace transforms, $F(k)$ can be determined to be $\mathrm{e}^{18}$

$$
\begin{aligned}
F(k) & =\frac{\sin \alpha \pi}{\pi} \frac{1}{k}\left(\frac{k}{k_{0}-k}\right)^{\alpha} k \leq k_{0} \\
& =0 \quad k>k_{0} .
\end{aligned}
$$

$F(k)$ is a normalized distribution function which is known as a beta distribution function. ${ }^{24}$ It is characterized by two discontinuities; one at $k=0$ and another $k=k_{0}$, where $k_{0}$ represents a maximum value of $k$.

A plot of $F(k)$ for various values of $\alpha$ appears in Fig. 7. It is apparent that the magnitude of $\alpha$ reflects the relative contributions of the more rapid and more slowly decaying contributions. The form of $F(k)$ suggests the solvent reorientation in glycol $-\mathrm{H}_{2} \mathrm{O}$ solvents is dominated by contribution of molecules in clusters which occur with a rate constant $k_{0}$, and clusters which are much more highly resistant to reorganization. The activation parameters determined from temperature-dependent studies then correspond to those associated with $k_{0}$. The smaller value of $\alpha$ for GW than for EGW reflects larger populations of molecular clusters or reorienting units in the former solvent which reorganize at the much slower rate.

The form of $F(k)$ given in Eq. (12) must be an approximation to the distribution of rate processes occurring within the system in that it predicts a significant population of solvent clusters which reorganize with an infinitely long reorientation time. The exciting wavelength dependence measurements both as a function of temperature and time reveal that the solvent completely reorganizes in a finite time. It is informative, however, to consider the system to be dominated by two solvent reorientation times or rate constants. The steady state solvent reorientation process which in general, if one assumes a distribution of rate constants, can be written as

$$
\frac{1}{\left(1+k_{0} \tau\right)^{\alpha}}=\sum_{k=0}^{\infty} \frac{F(k)}{1+k \tau}
$$

takes on the form

$$
\frac{1}{\left(1+k_{0} \tau\right)^{\alpha}} \cong \frac{a}{1+k_{0} \tau}+\frac{b}{1+k_{1} \tau},
$$

where $a$ and $b$ are the relative populations of solvent clusters which reorganize with rate constants $k_{0}$ and $k_{1}$. Values of $k_{0}$ and $k_{1}, a$ and $b$ can be found from the time experiments by fitting the experimental decay function $F(t, \tau)$ as the sum of only two exponentials. For proflavin in glycerol $-\mathrm{H}_{2} \mathrm{O}$ for which $\alpha=0.45$ the decay function at $T=156 \mathrm{~K}$ can be represented as

$$
F(t, T)=0.50 e^{-k_{0} t}+0.5 e^{-0.05 k_{0} t}
$$

in which $k_{0}=1 \mathrm{sec}^{-1}$ and $k_{1}=0.05 k_{0}$. Fitting the experimental steady state temperature-dependent data as the sum of two relaxation processes requires, however, knowledge of the activation parameters for the population of solvent clusters which reorganize with the smaller rate constant. In Fig. 8 the steady state solvent reorganization data from Fig. 3 for glycerol $-\mathrm{H}_{2} \mathrm{O}$ are represented as the sum of two contributions given by Eq. (15). A reasonable fit to the experimental data is achieved introducing the activation parameters for $k_{0}$ derived from the steady state analysis, and introducing values for the activation energy and entropy for $k_{1}$. The activation energy for the slower rate process is smaller, revealing that the population of solvent clusters of this type reorganizes more slowly as a consequence of a more highly unfavorable activation entropy.

Models for the structure of hydroxylic solvents have been advanced in which the occurrence of "ordered or structural" regions and of disordered regions has been emphasized. ${ }^{25-28}$ While the present data would seem 


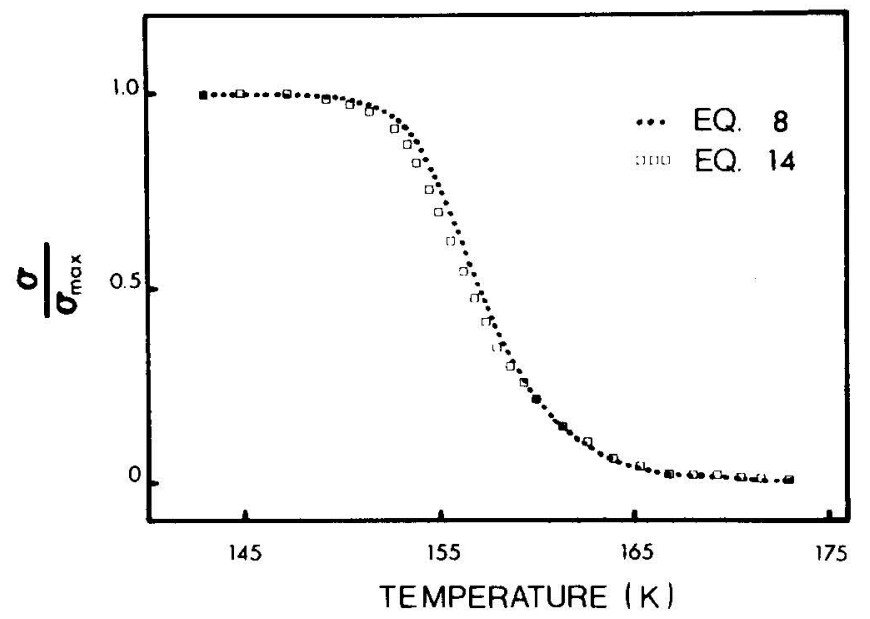

FIG. 8. A comparison of the empirical expression $\sigma / \sigma_{\max }$ $=\left[\left(1+k_{0} \tau\right)^{\alpha}\right]^{-1}$ employing the parameters found for $(3: 2)$ gly cerol-water; $(\bullet)$, with the behavior anticipated for the sum of only two reorientation rate constants given by Eq. (14); (a). Activation parameters for $k_{1}$ which yielded the best agreement were $E_{a}=20.7 \mathrm{kcal}$ and $\log A=28.2$ and were chosen by using a least squares procedure for $a=b=0.5$.

consistent with that notion, they do not provide a molecular basis for the bimodal distribution of rate processes. It should also be emphasized that nonexponential relaxation processes have been interpreted as evidence for a fundamentally cooperative proces $\mathrm{s}^{23,29,30}$ rather than for a distribution of relaxation processes. In subsequent publications the influence of perturbants for which there is evidence of an effect of water struc ture are examined with a view to elucidating the molecular basis for the nonexponential nature of solvent reorganization in hydroxylated solvents.

\section{APPENDIX}

\section{Correction for nonkinetic effects}

Experimental values of $\sigma$ were plotted as a function of temperature in the form $\log \sigma_{\max } / \sigma$ vs $T^{-1}\left(K^{-1}\right)$. At the cold end of the temperature scale these plots consistently showed a linear region which, unlike the principal transition, was independent of the excited-state lifetime of the chromophore employed. Substitution of glycerol$\mathrm{D}_{2} \mathrm{O}$ for glycerol $-\mathrm{H}_{2} \mathrm{O}$ also produced a marked shift in the principal transition but was without effect on the reduction in $\sigma$ in the cold temperature region. It was assumed that this nonkinetic contribution was linear throughout the principal transition. A straight line was drawn extrapolating from the cold end of the plot and the values of $\sigma / \sigma_{\max }$ at each temperature were then renormalized using this as a baseline.

\section{Determination of $\alpha, E_{a}$, and $A$}

Differentiation of Eq. (7) with respect to $T^{-1}$ yields

$$
d \log \left(\sigma_{\mathrm{max}} / \sigma\right) / d T^{-1}=-\alpha E_{a} k \tau / 2.303 R(1+k \tau),
$$

indicating that under conditions where $k \tau \gg 1$, a plot of $\log \left(\sigma_{\mathrm{max}} / \sigma-1\right)$ vs $T^{-1}$ is linear. The limiting slope yields $\alpha E_{a} / 2.303 R$, while the intercept becomes $\alpha \log A \tau$. The temperature at which $k_{0}=1 / \tau$ is equal to the ratio of the slope to the intercept. If $\phi$ denotes the value of $\log \left(\sigma_{\max } / \sigma\right)$ at $T_{k_{0}=1 / \tau}$, then $\alpha$ can be determined from the relation

$$
\alpha=3.32 \phi \text {. }
$$

It should be noted that according to Eq. (8) the slope of the line in Fig. 4 is equal to $E_{\alpha} / 2.303 R$. Thus over temperature ranges where the plot of $\log \tau$ vs $T_{k_{0}=1 / \tau}^{-1}$ is linear, $\alpha$ can be estimated as the ratio of the limiting slope of a $\log \sigma_{\max } / \sigma$ vs $T^{-1}$ plot to the slope of $\log \tau$ vs $T_{k_{0}=1 / \tau}^{-1}$ plot.

Failure to make the correction for the lifetime-independent part of transitions in $\sigma / \sigma_{\max }$ with temperature did not significantly alter the activation parameters $E_{a}$ and $\log A$ for the larger rate constant, $k_{0}$. The relative contribution of the slow and faster reorientation rate constants characterized by the parameter $\alpha$ were affected. In the absence of the correction the values of $\alpha$ lower by $20 \%-30 \%$, which had the effect of overemphasizing the contribution from the slow components.

${ }^{1}$ W. C. Galley and R. M. Purkey, Proc. Natl. Acad. Sci. USA 67, $1116(1970)$.

${ }^{2}$ A. N. Robinov and V. I. Tomin, Opt. Spectrose. 29, 578 (1970); 30, 461 (1971).

${ }^{3} \mathrm{~K}$. Itoh and T. Azumi, J. Chem. Phys, 62, 3431 (1975).

${ }^{4} \mathrm{H}$. H. Klingenberg and W. Rapp, Z. Phys. Chem. Neue Folge 84, 92 (1973).

${ }^{5}$ R. F. Chen, Anal. Biochem. 19, 374 (1967).

${ }^{6}$ A. N. Fletcher, J. Phys. Chem. 72, 2742 (1968).

${ }^{7}$ G. Weber and M. Shintzky, Proc. Natl. Acad. Sci. USA 65, 823 (1970).

${ }^{8}$ W. Klopfler, Chem. Phys. Lett. 11, 482 (1971).

${ }^{9}$ K. I. Rudik and L. G. Pikulik, Opt. Spectrosc. 20, 147 (1971).

${ }^{10}$ Yu. P. Kaulakis, G. P. Ionaitis, and A. T. Kazlauskene, Opt. Spectrosc. 33, 137 (1972).

${ }^{11}$ O. S. Khalil, C. J. Selinskar, and S. P. McGlynn, J. Chem. Phys, 58, 1607 (1973).

${ }^{12}$ R. M. Purkey and W. C. Galley, Biochemistry 9,3569 (1970).

${ }^{13}$ G. B. Strambini and W. C. Galley, Can. J. Spectrose. 21, 1-5 (1976).

${ }^{14}$ S. R. Anderson and G. Weber, J. Chem. Phys. 43, 139 (1969).

${ }^{15}$ M. S. Walker, T. W. Bednar, R. Lumry, and F. Humphries, Photobiol. 14, 147 (1971).

${ }^{16}$ R. F. Chen, G. G. Vurek, and N. Alexander, Science 158, 949 (1967)

${ }^{17}$ W. Kauzmann, Rev. Mod. Phys, 14, 12 (1942).

${ }^{18}$ Tables of Integral Transforms, edited by A. Erdelyi, W. Magnus, F. Oberhettinger, and F. G. Tricomi (McGraw-Hill, Toronto, 1954).

${ }^{19}$ N. G. Bakhshiev, Yu. T. Mazurenko, and I. V. Piterskaya, Opt. Spectrosc. 21, 307 (1966).

${ }^{20}$ N. G. Bakhshiev and J. V. Piterskaya, Opt. Spectrosc. 20, 437 (1968).

${ }^{21}$ W. R. Ware, P. Chow, and S. K. Lee, Chem. Phys. Lett. 2, 356 (1968).

${ }^{22} \mathrm{D}$. W. Davidson and R. H. Cole, J. Chem. Phys. 19, 1484 (1951).

${ }^{23}$ G. E. McDuffie, Jr., R. G. Quinn, and T. A. Litovitz, J. 
Chem. Phys. 37, 239 (1962).

${ }^{24}$ W. Feller, An Introduction to Probability Theory and Its $A P-$ plications (Wiley, Toronto, 1968), Vol. 1.

${ }^{25}$ H. S. Frank and W. Y. Wen, Discuss. Faraday Soc. 24, 133 (1945).

${ }^{26}$ G. Nemethy and H. A. Scheraga, J. Chem. Phys. 41, 680 (1974).
${ }^{27}$ A. Ben-Naim, J. Chem. Phys. 42, 1512 (1968).

${ }^{28}$ F. Franks, In Water: A Comprehensive Treatise, edited by F. Franks (Plenum, New York, 1973), Vol. 2, Chap. 1.

${ }^{29} \mathrm{G}$. E. McDuffie, Jr. and T. A. Litovitz, J. Chem. Phys. 37, 1699 (1962).

${ }^{30}$ T. A. Litovitz and G. E. McDuffie, Jr., J. Chem. Phys. 39, 729 (1963). 